\title{
Improvements to a Novel Device Simulating Pneumatic Intussusception Reduction
}

\author{
Steffan K. Soosman ${ }^{1}$, Joseph C. Li $^{2}$, Glade E. Roper ${ }^{3}$, Anthony S. Wexler ${ }^{4}$, \\ Rebecca Stein-Wexler ${ }^{3}$, Thomas Ray Sanchez ${ }^{3^{*}}$ \\ ${ }^{1}$ John A. Burns School of Medicine, University of Hawaii, Honolulu, USA \\ ${ }^{2}$ School of Medicine, University of California at Davis, Sacramento, USA \\ ${ }^{3}$ Department of Radiology, University of California at Davis, Sacramento, USA \\ ${ }^{4}$ Department of Mechanical and Aerospace Engineering, University of California at Davis, Civil and Environmental \\ Engineering, Land, Air, and Water Resources, Davis, USA \\ Email: *Thomas.sanchez@ucdmc.ucdavis.edu
}

Received December 7, 2012; revised March 1, 2013; accepted March 9, 2013

Copyright (C 2013 Steffan K. Soosman et al. This is an open access article distributed under the Creative Commons Attribution License, which permits unrestricted use, distribution, and reproduction in any medium, provided the original work is properly cited.

\begin{abstract}
Objectives: Intussusception, an uncommon but important cause of acute abdomen during infancy and early childhood, can be safely and effectively treated by fluoroscopic air reduction. Although pediatric radiology fellows report ample opportunity to practice intussusception reduction, radiology residents report few opportunities to participate in this procedure. Adequate training to reduce intussusception is essential, as successful reduction obviates the need for surgery. A teaching device that simulates intussusception reduction could help radiology residents develop the skills necessary to perform this procedure. Materials and Methods: We report on improvements made to a training device developed in 2010 by Stein-Wexler et al. Since then we have simplified the manufacturing process, added several unique patient cases, improved the software so that adding patient scenarios is easier, and improved the graphic interface to make the simulation more realistic and facilitate feedback. Results: The simulator is now a customizable and robust standalone package. A thorough instructor's manual and improvements to the graphic design-such as embedded checklists, built-in feedback mechanisms, and a more intuitive interface-make the simulator easier to use. Conclusion: We have improved our previously-reported teaching device for intussusception reduction and produced a robust simulator. We plan to make this device available to programs that train physicians in pneumatic intussusception reduction.
\end{abstract}

Keywords: Intussusception; Reduction; Simulator; Teaching

\section{Introduction}

Intussusception is an uncommon but important factor to consider in pediatric cases of acute abdomen. The incidence has been estimated at $0.5-2.3$ cases per 1000 live births in the USA [1,2]. First-line treatment for pediatric intussusception is enema reduction under the supervision of a radiologist. Both air and liquid enemas have been shown to be effective and safe [3], but air reduction is more likely to result in a successful reduction, causes less radiation exposure, and causes less peritoneal contamination in the event of perforation [4].

However, since intussusception is relatively uncommon, radiology residents have few opportunities to perform a supervised reduction. A survey found that senior radiology residents on average reported participating in

\footnotetext{
"Corresponding author.
}

only 2 cases during their entire residency; 22\% had been involved in no reductions, and 21\% had been involved only once [5]. A more recent survey found that pediatric radiology fellows have more experience with reducing intussusception, with respondents reporting an average of 6.9 reductions [6]. Meyer et al. [7] demonstrated that a radiologist must participate in at least 4 to 6 reductions to gain sufficient skill and confidence in this technique; by these criteria, the typical pediatric radiology fellow receives adequate training in intussusception reduction, but the typical radiology resident does not.

When a child presents with intussusception, a successful air reduction will obviate the need for surgery. It would be nice if all children could be treated at specialized pediatric hospitals, but many children with intussusception present at community hospitals that do not have pediatric radiologists on staff. It is therefore essen- 
tial that general radiologists be comfortable with this procedure.

In 2010, Stein-Wexler et al. [8] created a prototype for a training device that simulates the reduction procedure. Feedback about this device has led us to make improvements that transform it into a robust and versatile simulator that will be offered to programs that wish to supplement patient-based learning with a simulation model.

\section{Materials and Methods}

The authors sought to improve the prototype of the intussusception reduction simulator [8] with the goal of producing a device that other radiology training programs could purchase and use. The original design includes a custom machined polyvinyl chloride chamber and spring piston assembly that approximates the stress and strain properties of the colon. The trainee uses a standard aneroid bulb and gauge insufflator to pump air into the chamber. The pressure within the chamber is continuously monitored, and the software displays fluoroscopic still images of intussusception reduction when the trainee pushes a simulated fluoroscopic trigger.

We sought to improve the design of the simulator with the following goals:

1) Simplify the manufacturing process to reduce cost and complexity. Instead of using custom-machined parts, we tested modular and prefabricated materials that decrease cost per unit, decrease the risk of mechanical failure, and simplify maintenance.

2) Redesign the software to accommodate multiple patient scenarios. The original device included a single patient scenario, hard-coded into software, including clinical images, a stipulated ease of reduction, and the probability of perforation. We set out to redesign the program so that different patient scenarios could be added as independent "case files", each containing a unique patient presentation with associated clinical images. The ease of reduction and the probability of perforation are appropriate to the different clinical histories.

3) Provide feedback at multiple key stages for the trainee before and after the procedure. We include briefing and de-briefing stages, so the instructor has multiple opportunities for teaching and feedback, as well as the opportunity to review performance upon completion.

4) Improve the simulation experience by simplifying the graphical interface, adding the ability to stop the procedure and notify surgeons, and improving the quality of the fluoroscopic images. The original graphical interface was overly complex, and the user did not receive visual feedback that an image was taken. We set out to remove clutter from the screen and make the fluoroscopic image "flash" after every simulated exposure.

5) Create a comprehensive reference document for both the instructor and trainee.

This project study was IRB approved.

\section{Results}

We accomplished our goals as follows, resulting in a more robust and customizable training device:

1) The main pressure chamber of the original device was custom-machined from polyvinyl chloride, with ports drilled in it to allow for pressure sensing and air input. We found that a prefabricated 150 cc luer-lock syringe with a three-way stopcock attached worked just as well, and obviated the need to compromise the volume by drilling inlet ports. This modular approach to assembly saves considerable time and expense, and allows damaged or malfunctioning parts to be easily replaced.

2) The software was reprogrammed so that cases are no longer hard-coded in the program. The new version of the software is more flexible-each case is now a selfcontained file. When the user starts the program, it displays a list of the patients loaded onto the computer (Figure 1). The instructor or trainee chooses a patient from the list. Each case file includes the patient name, fluoroscopic images from an actual patient depicting different stages of reduction; pre-procedural and post-procedural images; and both abridged and detailed clinical presentation descriptions. Each case is customized to fit the corresponding clinical scenario, including the probability that the reduction will progress or retrogress and the probability of perforation at each stage of the reduction. It is now easy to add, delete, or modify cases in the case library, extending the utility of the simulator.

3) Pre-procedure and post-procedure stages were added to the simulator. The pre-procedure window (Figure 2) appears after the user selects a patient from the

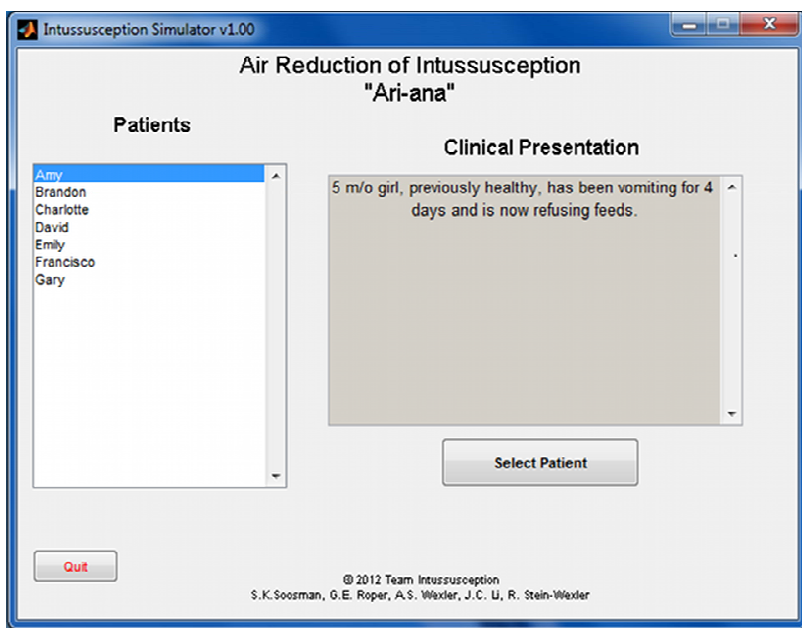

Figure 1. The case selection interface, or the "main menu". This interface appears upon starting the simulator. Patient cases are listed on the left, and an abridged clinical presentation is displayed for the highlighted patient. 


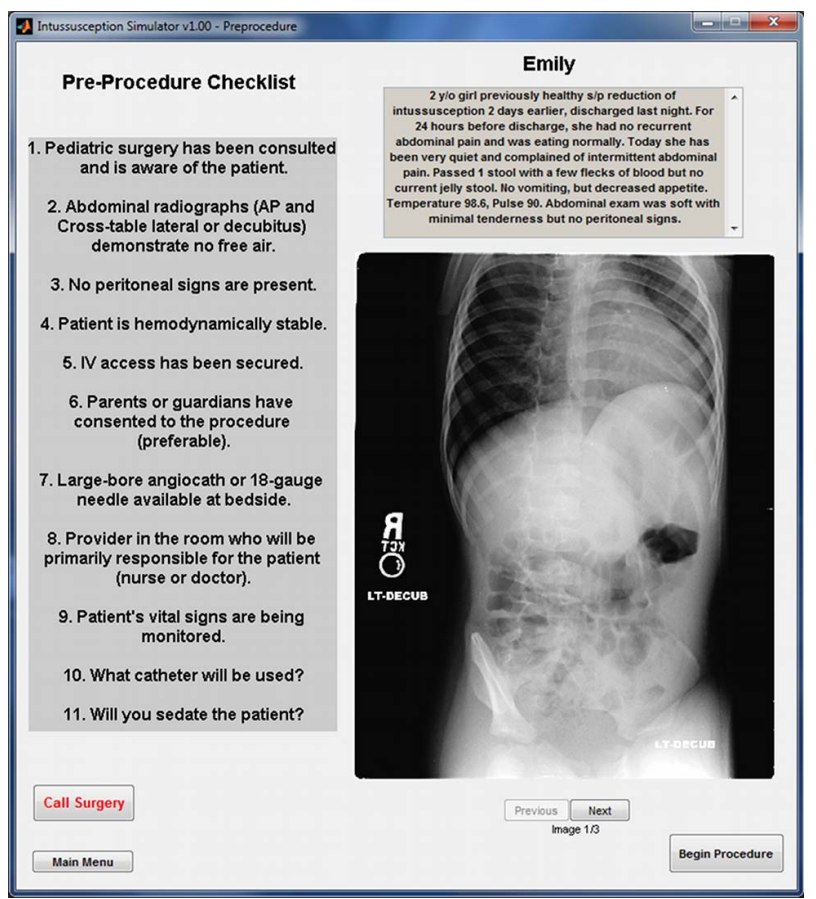

Figure 2. The pre-procedure interface. Scout radiographs, ultrasound and other pertinent images can be reviewed for the selected patient. A more detailed clinical presentation is also delineated at the top right. The embedded checklist on the left side of the interface reviews decision points and encourages discussion between the instructor and the trainee. main menu and includes a detailed clinical presentation and pre-procedure radiographs and/or ultrasound images. After reviewing the images, the trainee must decide whether to begin the procedure or-upon recognizing pre-existing pneumoperitoneum-to refer the patient to surgical services, terminating the scenario. In addition, a debriefing window now displays when the trainee exits each case (Figure 3). This post-procedural window presents an image of the last stage of reduction reached prior to termination, as well as a graph that plots the pressure within the system over the course of the simulation. If the intussusceptum is completely reduced and post-procedure radiographs are available, the trainee may review them. Post-procedure images are not offered if the reduction is incomplete, if they are not available for that specific patient, or if the bowel perforates.

4) We added a button to the screen that allows the trainee to terminate the procedure and refer to surgery at any time during the reduction. The trainee is expected to exercise this option if an image of pneumoperitoneum is displayed. The scenario immediately ends and the debriefing window is displayed, providing the opportunity for the instructor to review the case with the trainee. We also added more realistic image feedback-the screen flashes every time the trainee touches the simulated fluoroscopy button. This better mimics the functionality of the fluoroscope and provides the trainee with feedback that an image was taken, even if no significant progress

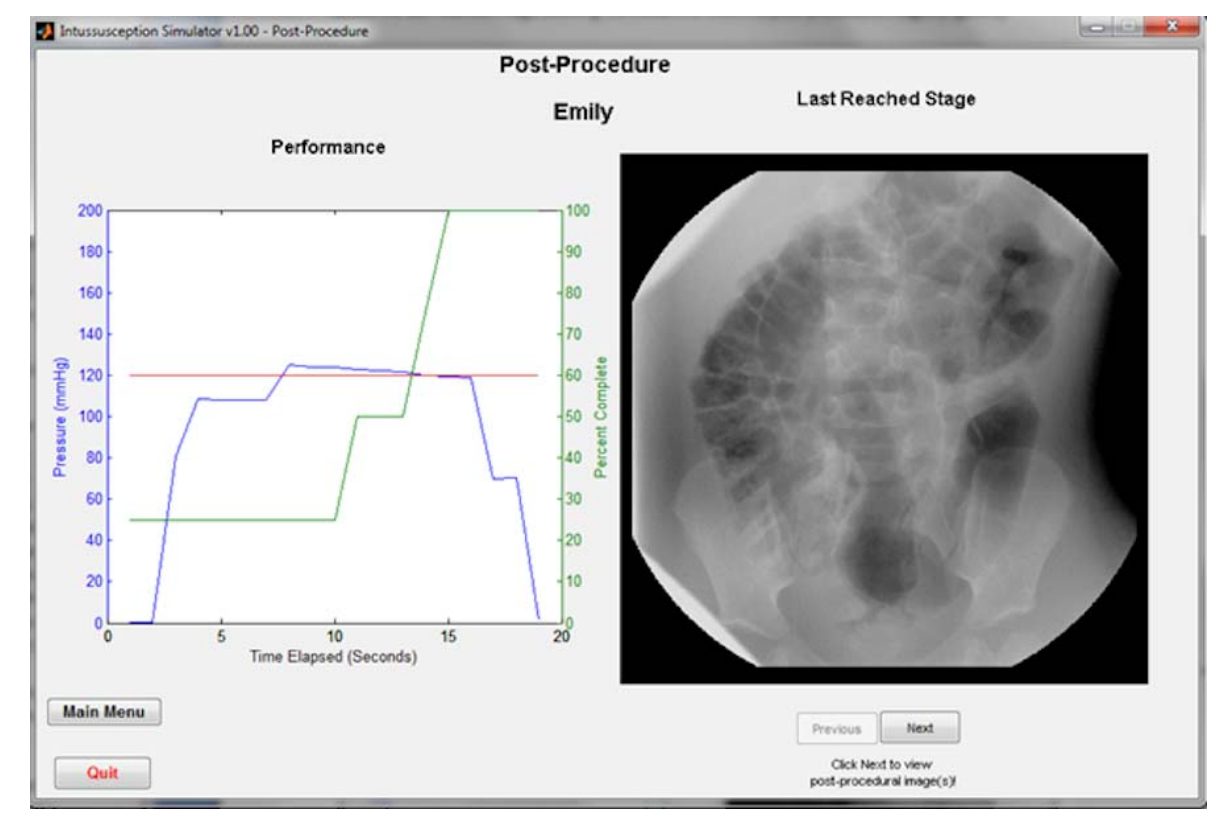

Figure 3. The post-procedure interface. Upon exiting the simulation, the post-procedure interface appears, providing feedback and the ability to review the last stage of reduction. The graph on the left utilizes two y axes. The left axis displays the pressure measured by the simulator in mmHg. The trainee's performance is graphed in blue, and the pressure of $120 \mathrm{mmHg}$ is delineated in red for reference and comparison. The right axis measures the trainee's progress throughout the simulation. The percentage complete at any given time is graphed in green. If the trainee completes the scenario, i.e., the green line reaches 100 percent, the option to review post-procedure images become available via the buttons below the "Last Reached Stage" image. This option will not appear if the percent complete is below $100 \%$. 
is made.

5) We have written a user's manual that includes information about intussusception and reduction, recommended reading, instructions for using the simulator, and initial setup procedures. We also include (for the instructor) a detailed description of each simulated patient that includes the clinical presentation, the ease of reduction, representative images from each reduction scenario with the salient features highlighted, and the predicted outcome of the reduction.

\section{Discussion}

Fluoroscopically guided hydrostatic intussusception reduction was one of the first interventional radiology procedures. First described by George M. Retan in 1927 [9], this method was popularized in Ravitch and McCune's 1948 experimental study [10]. Since then, fluoroscopically guided reduction has become the treatment of choice for uncomplicated cases of intussusception in childhood. While many centers outside of the United States use US to guide reductions [11,12], most pediatric radiologists in the United States and $90 \%$ of pediatric radiologists in European teaching hospitals prefer to reduce intussusceptions under fluoroscopic guidance [13].

Although the radiologist can use either air or liquid to reduce intussusception, reduction with air tends to cause smaller perforations and consequently less fecal peritoneal contamination [4]. Furthermore, the filling speed of air is approximately seven times faster than liquid, yielding a stronger force of reduction [14] and-according to some-increased probability of reduction [15]. It also exposes the patient to less radiation due to shorter fluoroscopic times and lower $\mathrm{kVp}$ and $\mathrm{mA}$ settings $[7,16]$. As a practical matter, air is less messy than liquid. For these reasons, pediatric radiologists increasingly favor a pneumatic approach [15,17]. We therefore built [8] and have now improved a simulator based on air reduction technique.

Due to the relatively low incidence of intussusception, radiology residents have little opportunity for supervised learning of the reduction procedure. In 2010, we developed our original device with the following goals in mind:

1) Include an air leak, forcing the trainee to recognize and address air loss and consequent pressure drops due to an inadequate seal.

2) Allow the reduction to proceed at variable levels of difficulty and speed depending to some degree on random chance, but mostly subject to the control of the instructor.

3) Present realistically challenging images to the trainee.

4) Have a higher-than-realistic likelihood of perfora- tion so that the trainee has the opportunity to recognize this complication and then practice treating the potentially fatal complication of tension pneumoperitoneum.

5) Encourage effective multi-tasking by requiring the trainee to simultaneously control the insufflator, monitor pressure, deliver radiation, interpret images, ensure a tight seal, and act quickly if perforation occurs.

With these improvements, the simulator is now a versatile and robust package. The transition to modular patient case files constitutes a major improvement. We have created seven different patients, and each offers a unique challenge to the trainee. For example, one patient has an intussusception that cannot be reduced, another is easily reduced, a third has no intussusception, and a fourth demonstrates free intraperitoneal air on the scout image. The detailed clinical presentation of each case may suggest the outcome of the procedure. However, although on average each case will tend to result in a certain outcome, the use of random number generators allows unexpected outcomes to occur as well, assuring that no two reductions of the same patient are exactly the same.

The original device was codenamed "LUCY" in homage to the toy doll that housed the pressure chamber. In light of the improvements and additional features made to LUCY, we have changed the simulator's name to ARI-ana, an acronym for Air Reduction of Intussusception.

This device has several limitations. First and foremost, using a simulator is inherently artificial. We found that instructor and trainee fatigue made it necessary to accelerate the entire experience, compressing a sometimes lengthy procedure into just a few minutes. Furthermore, as described above, the probability of a perforation during the reduction is, by design, higher with this simulator than in real life. In addition, this device depends on the educational interaction between instructor and trainee-it is designed to supplement rather than replace the experience of reducing an intussusception in a real patient.

\section{Conclusion}

Air reduction is a safe and effective treatment for intussusception, but the technique requires practice. Children will benefit if radiologists in the community are comfortable with the air reduction technique since it avoids surgical intervention. Since opportunities to reduce intussusception during residency are limited, an educational device that simulates air reduction could supplement radiology residents' training in intussusception reduction. We have created such a device, and have since refined it into a robust, versatile simulator. We hope to make this simulator available for use by other radiology programs. 


\section{REFERENCES}

[1] J. Bruce, Y. S. Huh, D. R. Cooney, M. P. Karp, J. E. Allen and T. C. Jewett Jr., "Intussusception: Evolution of Current Management," Journal of Pediatric Gastroenterology and Nutrition, Vol. 6, No. 5, 1987, pp. 663-674. doi:10.1097/00005176-198709000-00003

[2] M. B. Rennels, U. D. Parashar, R. C. Holman, C. T. Le, H. G. Chang and R. I. Glass, "Lack of an Apparent Association between Intussusception and Wild or Vaccine Rotavirus Infection," Pediatric Infectious Disease Journal, Vol. 17, No. 10, 1998, pp. 924-925. doi:10.1097/00006454-199810000-00018

[3] S. W. Beasley and N. A. Myers, "Intussusception: Current Views,” Pediatric Surgery International. Vol. 14, No. 3, 1998, p.157. doi:10.1007/s003830050473

[4] A. Danerman, D. J. Alton, S. Ein, D. Wesson, R. Superina and P. Thorner, "Perforation during Attempted Intussusception Reduction in Children-A Comparison of Perforation with Barium and Air," Pediatric Radiology, Vol. 25, No. 2, 1995, pp. 81-88. doi:10.1007/BF02010311

[5] C. Bateni, R. Stein-Wexler, S. L. Wootton-Gorges and C. S. Li, "Radiology Residents' Experience with Intussusception Reduction,” Pediatric Radiology, Vol. 41, No. 6, 2011, pp. 21-26. doi:10.1007/s00247-010-1923-0

[6] R. Stein-Wexler, C. Bateni, S. L. Wootton-Gorges, C. S. Li, "Pediatric Radiology Fellows' Experience with intussusception Reduction,” Pediatric Radiology, Vol. 41, No. 11, 2011, pp. 1365-1368. doi:10.1007/s00247-011-2095-2

[7] J. S. Meyer, B. C. Dangman, C. Buonomo and J. A. Berlin, "Air and Liquid Contrast Agents in the Management of Intussusception: A Controlled, Randomized Trial," Radiology, Vol. 188, No. 2, 1993, pp. 507-511.

[8] R. Stein-Wexler, T. Sanchez, G. E. Roper, et al., “An Interactive Teaching Device Simulating Intussusception Reduction,” Pediatric Radiology, Vol. 40, No. 11, 2010, pp. 1810-1815. doi:10.1007/s00247-010-1764-X

[9] V. G. McDermott, "Childhood Intussusception and Ap- proaches to Treatment: A Historical Review,” Pediatric Radiology, Vol. 24, No. 3, 1994, pp. 153-155. doi:10.1007/BF02012174

[10] M. M. Ravitch and C. R. McCune, "Reduction of Intussusception by Hydrostatic Pressure; An Experimental Study," Bulletin of the Johns Hopkins Hospital, Vol. 82, No. 5, 1948, pp. 550-568.

[11] T. W. Riebel, R. Nasir and K. Weber, "US-Guided Hydrostatic Reduction of Intussusception in Children," $R a$ diology, Vol. 188, No. 2, 1993, pp. 513-516.

[12] G. D. Wang and S. J. Liu, "Enema Reduction of Intussusception by Hydrostatic Pressue under Ultrasound Guidance: A Report of 377 Cases,” Journal of Pedatric Surgery, Vol. 23, No. 9, 1988, pp. 814-818. doi:10.1016/S0022-3468(88)80229-X

[13] P. Schmit, W. K. Rohrschneider and D. Christmann, "Intestinal Intussusception Survey about Diagnostic and Nonsurgical Therapeutic Procedures,” Pediatric Radiology, Vol. 29, No. 10, 1999, pp. 752-761. doi:10.1007/s002470050689

[14] T. Schmitz-Rode, C. Müller-Leisse and G. Alzen, “Comparative Examination of Various Rectal Tubes and Contrast Media for the Reduction of Intussusceptions," Pediatric Radiology, Vol. 21, No. 5, 1991, pp. 341-345. doi:10.1007/BF02011482

[15] A. Daneman and O. Navarro, "Intussusception Part 2: An Update on the Evolution of Management," Pediatric Radiology, Vol. 34, No. 2, 2004, pp. 97-108. doi:10.1007/s00247-003-1082-7

[16] E. Phelan, J. F. de Campo and G. Malecky, “Comparison of Oxygen and Barium Reduction of Ileocolic Intussusception,” American Journal of Roentgenolog , Vol. 150, No. 6, 1988, pp. 1349-1352. doi:10.2214/ajr.150.6.1349

[17] J. S. Meyer, "The Current Radiologic Management of Intussusception: A Survey and Review,” Pediatric Radiology, Vol. 22, No. 5, 1992, pp. 323-325. doi:10.1007/BF02016244 